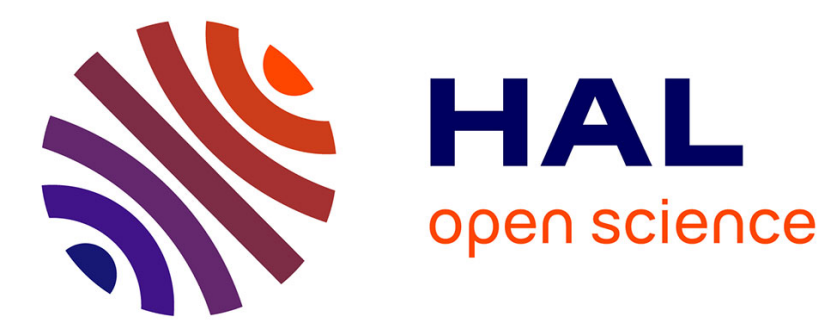

\title{
Thermomagnetically patterned micromagnets
}

Frédéric Dumas-Bouchiat, Luiz-Fernando Zanini, S. Kustov, Nora Dempsey, R. Grechishkin, Klaus Hasselbach, Jean-Christophe Orlianges, Corinne Champeaux, Alain Catherinot, Dominique Givord

\section{To cite this version:}

Frédéric Dumas-Bouchiat, Luiz-Fernando Zanini, S. Kustov, Nora Dempsey, R. Grechishkin, et al.. Thermomagnetically patterned micromagnets. Applied Physics Letters, 2010, 96 (10), pp.102511. 10.1063/1.3341190 . hal-00569295

\section{HAL Id: hal-00569295 \\ https://hal.science/hal-00569295}

Submitted on 28 Oct 2021

HAL is a multi-disciplinary open access archive for the deposit and dissemination of scientific research documents, whether they are published or not. The documents may come from teaching and research institutions in France or abroad, or from public or private research centers.
L'archive ouverte pluridisciplinaire HAL, est destinée au dépôt et à la diffusion de documents scientifiques de niveau recherche, publiés ou non, émanant des établissements d'enseignement et de recherche français ou étrangers, des laboratoires publics ou privés.

\section{(c)(1)}

Distributed under a Creative Commons Attribution| 4.0 International License 


\title{
Thermomagnetically patterned micromagnets
}

\author{
F. Dumas-Bouchiat, ${ }^{1, a)}$ L. F. Zanini, ${ }^{1}$ M. Kustov, ${ }^{1,2}$ N. M. Dempsey, ${ }^{1}$ R. Grechishkin, ${ }^{3}$ \\ K. Hasselbach, ${ }^{1}$ J. C. Orlianges, ${ }^{4}$ C. Champeaux, ${ }^{4}$ A. Catherinot, ${ }^{4}$ and D. Givord ${ }^{1}$ \\ ${ }^{1}$ Institut Néel, CNRS, Université Joseph Fourier, 25 rue des Martyrs 38042 Grenoble, France \\ ${ }^{2}$ G2Elab Laboratoire de Génie Electrique, INP de Grenoble, 961 rue de la Houille Bianche, \\ 38042 St-Martin-d'Héres, France \\ ${ }^{3}$ Laboratory of Magnetoelectronics, Tver State University, 170043 Tver, Russia \\ ${ }^{4}$ SPCTS, CNRS, Université de Limoges, 123 Avenue A. Thomas, 87060 Limoges, France
}

Thermomagnetic patterning (TMP) of $4 \mu \mathrm{m}$ thick high performance NdFeB hard magnetic films deposited on Si substrates has been achieved using single pulsed laser irradiation. Uniaxially magnetised chessboard and stripe patterns with lateral feature sizes in the range 50-100 $\mu \mathrm{m}$ were produced. The depth of reversal was estimated, using both global (vibrating sample magnetometry) and localized (scanning Hall probe) measurements, to be in the range of 1.1-1.2 $\mu \mathrm{m}$. A simple model provides semiquantitative agreement with the experimental results. A linear Halbach array was fabricated to demonstrate the potential of TMP for the realization of complex multidirectional microflux sources.

Microflux sources produce magnetic fields which are spatially modulated at the micron scale. They have many potential applications in the field of magnetic microelectromechanical systems, ${ }^{1}$ as well as for biasing, ${ }^{2}$ diamagnetic levitation, ${ }^{3}$ the manipulation and trapping of particles and atoms, ${ }^{4,5}$ etc. The use of permanent magnets to produce the flux favors autonomy and stability while down-scaling the size of the magnet does not lead to a reduction in the flux density achievable from a given material. ${ }^{1}$ The challenge in fabricating permanent magnet based sources is to produce hard magnetic films of the appropriate thickness $(1-100 \mu \mathrm{m})$, and to laterally pattern them on the scale of 1-100 $\mu \mathrm{m}$. Physical vapor deposition techniques have been shown to be suitable for the preparation of high quality rare earth transition metal magnets in film form. ${ }^{6-10}$ Lateral patterning of such films using established techniques (lithography and etching of the substrate and/or the film) requires a number of microfabrication steps and is complicated by the reactive nature of the rare earth components. ${ }^{11-13}$ In this paper, we report on the use of thermomagnetic patterning (TMP) to produce micromagnets, which serve as microflux sources to produce spatially modulated stray fields. This patterning technique is relatively simple, requiring no topographic patterning of the hard magnetic film, and higher stray field values may be expected as no magnetic material is removed. The basic idea behind the approach is to exploit the temperature dependence of coercivity ${ }^{14}$ to switch magnetization. This idea has already been used in various contexts, in general to achieve bit by bit writing ${ }^{15,16}$ or to produce millimeter-sized surface patterns in bulk magnets via irradiation through a mask. ${ }^{17}$ The specificity of the approach described in this paper is to propose a method for the patterning of integratable hard magnetic films at the micron scale.

High rate triode sputtering was used to deposit $4 \mu \mathrm{m}$ thick NdFeB films, which are either out-of-plane (oop) textured or isotropic, on $100 \mathrm{~mm} \mathrm{Si}$ wafers. ${ }^{9}$ Ta $(100 \mathrm{~nm})$ was used as both a buffer and capping layer to prevent diffusion

${ }^{a)}$ Electronic mail: frederic.dumas-bouchiat@ grenoble.cnrs.fr. into the $\mathrm{Si}$ and oxidation, respectively. At $300 \mathrm{~K}$, The films have coercive field $\mu_{0} H_{c}(300)$ values of about $1.9 \mathrm{~T}$ and remanent magnetization values close to 1.4 and $0.7 \mathrm{~T}$ for the anisotropic and isotropic films, respectively. Masks for TMP were prepared by lithographic patterning of $2 \times 2 \mathrm{~mm}^{2} \mathrm{Cr}$ films deposited on $5 \times 5 \mathrm{~mm}^{2}$ quartz substrates of thickness $525 \mu \mathrm{m}$. The masks consisted of chessboard and stripe patterns with characteristic dimensions in the range $50-100 \mu \mathrm{m}$. The films were irradiated through a mask with a $\mathrm{KrF}(248 \mathrm{~nm})$ pulsed excimer laser during $25 \mathrm{~ns}$ in a magnetic field of $0.5 \mathrm{~T}$, produced by a bulk permanent magnet. The maximum laser fluence was fixed at $240 \mathrm{~mJ} / \mathrm{cm}^{2}$. Higher fluences damage the film surface. Uniaxial magneto optic indicator films (U-MOIF) and planar (P-MOIF) magneto optic indicator films ${ }^{18}$ were used for qualitative characterization of the patterned $\mathrm{NdFeB}$ films.

The TMP process is schematized in Fig. 1(a). The hard magnetic film to be patterned is initially saturated in one direction (step 1). The film is then locally heated by irradiation through a mask. During irradiation, a field is applied of opposite direction to that of the magnetization, the strength of which $\mu_{0} H_{\text {app }}$ is such that the total field, $\mu_{0} H_{\text {tot }}$ (applied field+demagnetizing field), acting on the magnetic film is less than $\mu_{0} H_{c}(300)$ (step 2). The temperature at the film's surface rises quickly and then heat diffuses through the film (step 3). In the regions heated up to a temperature $T$ such that $\mu_{0} H_{\text {tot }}$ overcomes $\mu_{0} H_{c}(T)$ switching of the magnetization occurs. The resultant structure consists of an array of oppositely magnetized micromagnets (step 4).

According to the schematic diagram in Fig. 1(b), a U-MOIF can produce binary black and white images. The U-MOIF images shown in Figs. 1(c) and 1(d) demonstrate the applicability of TMP to produce an array of alternatively magnetized micromagnets. We clearly see the chessboard magnetic pattern produced above the section of the oop textured film covered by the Cr mask, each square corresponding to a micromagnet of surface area $50 \times 50 \mu \mathrm{m}^{2}$. Striped features of approximate width $33 \mu \mathrm{m}$ due to light interferences are observed outside the chessboard mask. Indeed, the top and bottom quartz surfaces are not perfectly parallel and 
(a)

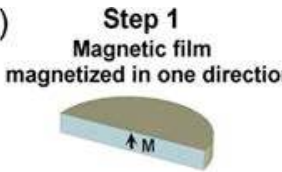

Step 2

Nanosecond pulsed laser irradiation under reverse external magnetic field
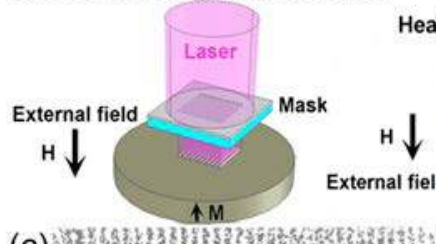

Heat diffusion through the film

(c)

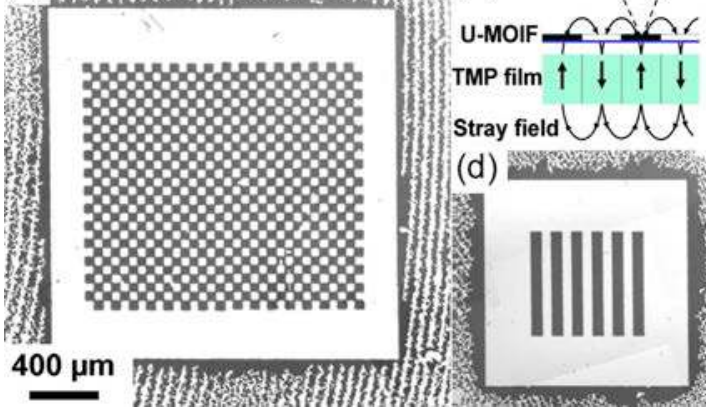

FIG. 1. (Color online) [(a) and (b)] Schematic diagrams of the TMP process and magneto-optic imaging with an indicator film. [(c) and (d)] Magnetooptic images of a U-MOIF placed on top of TMP out of plane textured $\mathrm{NdFeB}$ films prepared using a chessboard mask (square $50 \times 50 \mu \mathrm{m}^{2}$ ) and a stripe mask (stripe width $100 \mu \mathrm{m}$ ).

magnetization reversal occurs where interference leads to maximum light intensity, and thus to significant heating. Such interference patterning could be used to produce magnetic patterns which are much smaller than the feature size of the mask used. They are not discussed further in the present paper.

Two methods were used to estimate the depth of film over which magnetization had been reversed by TMP. The first method is based on macroscopic measurements using a vibrating sample magnetometer (VSM). The remanent magnetization of the as-irradiated film $\left(M_{r i}\right)$ is compared with the remanent magnetization measured following saturation in a field of $8 \mathrm{~T}\left(M_{r s}\right)$ (Fig. 2-inset).

The reversal depth, $d$, in a film of thickness, $t$, was then derived from the ratio of the irradiated surface area $\left(S_{i}\right)$, and the nonirradiated one $\left(S_{n i}\right)$, itself deduced from U-MOIF observations, according to the following expression (1):

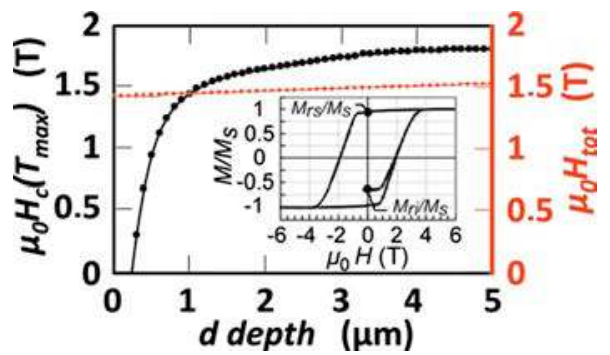

FIG. 2. (Color online) $\mu_{0} H_{c}\left(T_{\max }\right)$ and $\mu_{0} H_{\text {tot }}$ vs depth. The point of intersection gives the depth $d$ of magnetization reversal. Inset: hysteresis loop (without demagnetizing field correction) of a $4 \mu \mathrm{m}-\mathrm{NdFeB}$ film $\left(\mu_{0} H_{c}\right.$ $=1.9 \mathrm{~T})$ showing the remanence of an irradiated $\left(M_{r i} / M_{s}\right)$ and then resaturated $\left(M_{r s} / M_{s}\right)$ film.

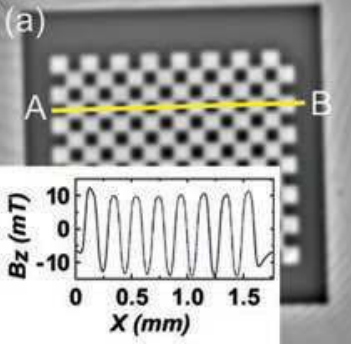

(b)

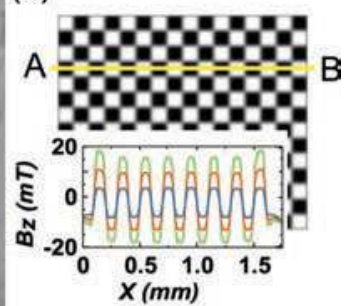

FIG. 3. (Color) (a) Hall probe measurement of $B_{z}$ distribution (graduations of gray) produced at $26 \mu \mathrm{m}$ above a TMP film; inset $-B_{z}$ profile along the line $\mathrm{AB}$. (b) Calculated pattern and $B_{z}$ profile along $\mathrm{AB}$ for magnetization reversal depths [0.5 (blue), 1 (red) and (green) $1.5 \mu \mathrm{m}]$.

$$
d=\frac{1}{2} t\left(\frac{S_{i}+S_{n i}}{S_{i}}\right)\left(1 \pm \frac{M_{r i}}{M_{r s}}\right) .
$$

In this simple model, the reversed volume is taken to be a parallelepiped and it is assumed that in both the reversed and non-reversed regions, the magnetization is equal to $M_{r s}$. Using this macroscopic approach, we estimated a reversal depth of $1.2 \pm 0.3 \mu \mathrm{m}$. The error here is essentially determined by the uncertainty in the estimation of the film thickness and the ratio of the volume of irradiated and nonirradiated zones.

The second approach used to estimate $d$ is based on a localized measurement of the $z$-component of the stray field produced by the patterned films, using a scanning Hall probe microscope (SHPM). ${ }^{19}$ The Hall probe was scanned at a height of approximately $26 \mu \mathrm{m}$ above the surface of the film and the measured $z$-component of the stray field at this height is plotted in Fig. 3(a).

This measurement reveals the expected chessboard pattern of alternatively magnetized square domains (in this particular case the domains have a size of $100 \times 100 \mu \mathrm{m}^{2}$ ). The measured average peak-to-peak magnetic field value is $24 \mathrm{mT}$. Assuming a remanent magnetization of $1.4 \mathrm{~T}$ for both the reversed and non-reversed domains the produced field was calculated at a height of $26 \mu \mathrm{m}$ for different $d$ [Fig. 3(b)]. Peak-to-peak values of magnetic field of 22, 45, and $87 \mathrm{mT}$ were found for $d$ of 1,2 , and $4 \mu \mathrm{m}$, respectively. Comparing these values to those measured experimentally, $d$ is estimated to be $1.1 \pm 0.2 \mu \mathrm{m}$. The error here associates the uncertainty in the estimation of the reversed volume and the probe to sample distance. Within experimental accuracy, $d$ obtained by the two methods are in fair agreement.

In the following discussion, we take into account that all TMP NdFeB films presented here and prepared by triode sputtering present a granular microstructure. ${ }^{9}$ For simplicity, we assume that all grains have the same coercive field value $\mu_{0} H_{c}(T)$. The condition for magnetization reversal of a given grain, located at depth $d$, is that the total field acting on this grain, $\mu_{0} H_{\text {tot }}(d)$, be larger than $\mu_{0} H_{c}\left[T_{\max }(d)\right]$ where $T_{\max }(d)$ is the maximum temperature reached at depth $d$. Considering that the energy in a given single pulse is equal to $240 \mathrm{~mJ}$, $T_{\max }(d)$ was evaluated using the classical heat diffusion equation. ${ }^{14}$ The field $\mu_{0} H_{c}\left[T_{\max }(d)\right]$, directly derived from $\mu_{0} H_{c}(T)$ measurements performed before film irradiation, is plotted in Fig. 2(a). $H_{\text {tot }}(d)$ is equal to $H_{\text {ext }}+H_{\text {demag }}(d)$, where $H_{\text {ext }}$ is the field generated by the biasing permanent magnet placed beneath the film $\left(\mu_{0} H_{\text {ext }}=0.5 \mathrm{~T}\right)$ and $H_{\text {demag }}(d)$ is the demagnetizing field created by the film itself. Initially, the film magnetization is $\mu_{0} M_{r}=1.4 \mathrm{~T}$. $H_{\text {demag }}(0)$ is given by 


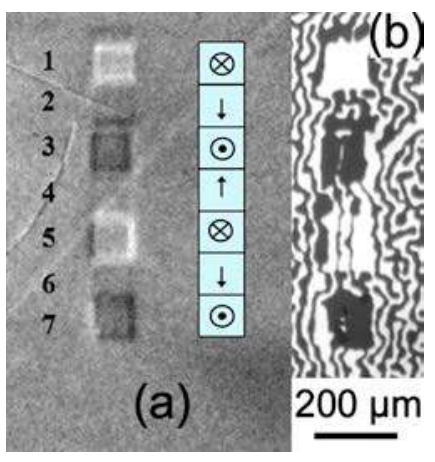

FIG. 4. (Color online) (a) Magneto-optic image of a P-MOIF placed on top of a thermomagnetically patterned Halbach array and schematic diagram showing the direction of the applied field used during TMP. (b) Magnetooptic image of the same array made using a U-MOIF.

$\left(1-N_{g}\right) M_{r}$ where $N_{g}$ is the demagnetizing field factor of an individual grain (equal to $1 / 3$ for spherical grains). ${ }^{20}$ Thus $\mu_{0} H_{\text {demag }}(0) \approx 0.9 \mathrm{~T}$ and $\mu_{0} H_{\text {tot }}(0)=1.4 \mathrm{~T}$. After the magnetization in a region of depth $d$ has been reversed, the film contains two following distinct regions: (i) a lower saturated region, of thickness $(t-d)$, source of a demagnetizing field equal to $0.9 \mathrm{~T}$, as above, and (ii) an upper patterned region of thickness $d$, the contribution of which to the demagnetizing field was calculated numerically. The total field, $\mu_{0} H_{\text {tot }}(d)$ thus derived at the center of each individual irradiated zone is plotted in Fig. 2(a). The two curves, representative of the functions $\mu_{0} H_{c}\left[T_{\max }(d)\right]$ and $\mu_{0} H_{\text {tot }}(d)$, respectively, intersect at $d_{0}=1.1 \mu \mathrm{m}$. Up to $d=d_{0}$, the total field being larger than the coercive field, the grain magnetization is reversed whereas for $d>d_{0}$, the coercive field is larger than $\mu_{0} H_{\text {tot }}(d)$ so that reversal cannot be nucleated. Considering that after nucleation, magnetization reversal propagates throughout the concerned grain, statistically the expected reversed region depth is $d_{\max }=d_{0}+D_{\text {grain }} / 2$, where $D_{\text {grain }}$ is the average grain diameter. From SEM observation, $D_{\text {grain }} \approx 0.4 \mu \mathrm{m}$, so that $d_{\max }=1.3 \mu \mathrm{m}$, which compares favorably with the experimentally determined value of $1.1-1.2 \mu \mathrm{m}$.

Specific arrangements of the relative direction of magnetization of an assembly of permanent magnets can be used to produce complex magnetic field configurations including one-sided flux patterns $\mathrm{s}^{21}$ and linear Halbach arrays and Halbach cylinders. ${ }^{22}$ The former arrangement maximizes the flux produced on one side of the arrangement and minimizes it on the other, the latter produces an intense magnetic field confined entirely within the cylinder with zero field outside. At the macroscopic scale, such structures are produced by assembling premagnetized magnets or using complex magnetizing fixtures based on pulsed electromagnets. Neither of these approaches is feasible at the micron scale. We chose to prepare a linear Halbach array, to assess the suitability of TMP to prepare complex patterns. Seven successive irradiations were applied to a $4 \mu \mathrm{m}$ thick isotropic $\mathrm{NdFeB}$ film, through a mask containing a single hole $\left(100 \times 100 \mu \mathrm{m}^{2}\right)$. Between each irradiation, the mask was moved by $100 \mu \mathrm{m}$ along a straight line and the direction of applied magnetic field was rotated by $\pi / 2$. Magneto-optic images, made using both U and P-MOIFs [Figs. 4(a) and 4(b)], clearly demonstrate that the micromagnets labeled 1, 3, 5, and 7 are magnetized in the oop direction, sections 1 and 5 being magnetized opposite to sections 3 and 7 . In the case of the P-MOIF image, the black/white contrast is sharpest at the feature edges, where the field strength is greatest. Both the nonsaturated state of the U-MOIF and the gray contrast in the P-MOIF above micromagnets 2,4 , and 6 , indicate the absence of an oop field component above these magnets. These images qualitatively demonstrate the possibility to produce complex, multidirectional patterns at the micron-scale using TMP. Note that the strength of field profiles produced by the structure presented here is limited by the relatively large lateral size of the features produced $\left(100 \times 100 \mu \mathrm{m}^{2}\right)$ compared to the depth of magnetization reversal achieved (of the order of $1 \mu \mathrm{m}$ ), owing to demagnetizing field effects. A quantitative analysis of Halbach arrays with finer lateral feature sizes is underway.

In conclusion, we have demonstrated that TMP is suited to the fabrication of microflux sources using hard magnetic films. Magnetization switching to depths of 1.1-1.2 $\mu \mathrm{m}$ was achieved. Both uniaxial and multiaxial structures have been prepared and are presently being tested for the levitation of diamagnetic objects and the trapping of ferromagnetic objects.

The authors acknowledge support from the RTRA Fondation Nanosciences (M.K.) and the Federal Special Purpose Program of the Russian Agency for Education (R.G.) and would like to thank D. Hykel and P. Laczkowski for their help in setting up the SHPM.

${ }^{1}$ O. Cugat, J. Delamare, and G. Reyne, IEEE Trans. Magn. 39, 3607 (2003).

${ }^{2}$ V. Neu, A. Anane, S. Wirth, P. Xiong, S. A. Shaheen, and F. J. Cadieu, J. Appl. Phys. 87, 5350 (2000).

${ }^{3}$ H. Chetouani, V. Haguet, C. Jeandey, C. Pigot, A. Walther, N. M. Dempsey, F. Chatelain, B. Delinchant, and G. Reyne, Solid-State Sensors, Actuators and Microsystems Conf., Transducers 07, 715 (2007).

${ }^{4}$ P. Kauffmann, H. Chetouani, P. Pham, V. Haguet, and G. Reyne, Sens. Lett. 7, 470 (2009).

${ }^{5}$ Y. T. Xing, I. Barb, R. Gerritsma, R. J. C. Spreeuw, H. Luigjes, Q. F. Xiao, C. Rétif, and J. B. Goedkoop, J. Magn. Magn. Mater. 313, 192 (2007).

${ }^{6}$ F. J. Cadieu, in Physics of Thin Films, edited by M. Francombe and J. Vossen (Academic, New York, 1992), Vol. 16.

${ }^{7}$ B. A. Kapitanov, N. V. Kornilov, Y. L. Linetsky, and V. Y. Tsvetkov, J. Magn. Magn. Mater. 127, 289 (1993).

${ }^{8}$ A. Nakano, R. Kato, H. Fukunaga, and F. Yamashita, IEEE Trans. Magn. 38, 2913 (2002).

${ }^{9}$ N. M. Dempsey, A. Walther, F. May, D. Givord, K. Khlopkov, and O. Gutfleisch, Appl. Phys. Lett. 90, 092509 (2007).

${ }^{10}$ A. Walther, D. Givord, N. M. Dempsey, K. Khlopkov, and O. Gutfleisch, J. Appl. Phys. 103, 043911 (2008).

${ }^{11}$ J. J. Wang, J. R. Childress, S. J. Pearton, F. Sharifi, K. H. Dabmen, E. S. Giliman, F. J. Cadieu, R. Rani, X. R. Qian, and L. Chen, J. Electrochem. Soc. 145, 2512 (1998)

${ }^{12}$ T. Budde and H. H. Gatzen, J. Appl. Phys. 99, 08 N304 (2006).

${ }^{13}$ A. Walther, C. Marcoux, B. Desloges, R. Grechishkin, D. Givord, and N. M. Dempsey, J. Magn. Magn. Mater. 321, 590 (2009).

${ }^{14}$ See supplementary material at http://dx.doi.org/10.1063/1.3341190 for the graph $\mu_{0} H_{c}$ vs $T$ and the heat diffusion model details.

${ }^{15}$ L. Mayer, J. Appl. Phys. 29, 1454 (1958).

${ }^{16} \mathrm{P}$. Hansen, J. Magn. Magn. Mater. 83, 6 (1990).

${ }^{17}$ J. R. Bradley, T. A. Perry, and T. Schroeder, U.S. Patent No. 5283130 (February 1, 1994).

${ }^{18}$ R. M. Grechishkin, S. Chigirinsky, M. Gusev, O. Cugat, and N. M. Dempsey, in Magnetic Nanostructures in Modern Technology, edited by B. Azzerboni, G. Asti, L. Pareti, and M. Ghidini (Springer-Kluwer Academic, Dordrecht, 2007), p. 195.

${ }^{19}$ The quantum well hall sensors (QWHS) based on AlGaAs/InGaAs/GaAs heterostructures were provided by V. Mosser at Itron SAS, France.

${ }^{20}$ A. N. Dobrynin, V. M. T. S. Barthem, and D. Givord, Appl. Phys. Lett. 95, 052511 (2009).

${ }^{21}$ J. C. Mallinson, IEEE Trans. Magn. 17, 2453 (1981).

${ }^{22}$ K. Halbach, Nucl. Instrum. Methods 169, 1 (1980). 